\title{
Time-resolved photoacoustic spectroscopy using fiber Bragg grating acoustic transducers
}

\author{
Qingxin Yang ${ }^{\text {a }}$, Jack Barnes ${ }^{\text {a }}$, Hans-Peter Loock ${ }^{\mathrm{a}, *}$, David Pedersen ${ }^{\mathrm{b}}$ \\ a Department of Chemistry, Queen's University, Kingston, Ont., Canada, K7L $3 N 6$ \\ ${ }^{\mathrm{b}}$ Defence Research and Development Canada, Suffield, Medicine Hat, Alta., Canada, T1B 8 K6
}

Received 28 July 2006; received in revised form 8 February 2007; accepted 29 March 2007

\begin{abstract}
An acoustic transducer based on a fiber Bragg grating (FBG) is presented and characterized for use in time-resolved laser-induced photoacoustic spectroscopy (PAS) on solid samples. The photoacoustic wave was generated by pulsed laser excitation of immobilized carbon black or erbium oxide powder, and detected by recording either the transmission or reflection spectrum of a clamped fiber Bragg grating (FBG). The characterization of the FBGs photoacoustic response is based on the experimental comparison with a static lateral strain source and on theoretical analysis using the piecewise-uniform approach to photoelastic theory. The temporal resolution of the response is determined by the arrangement of the FBG with respect to the source of the acoustic wave and is better than $150 \mathrm{~ns}$, as was verified in a deconvolution analysis.

The method has not only a fast time response but also is simple, inexpensive, and accurate as indicated by the good agreement of the experimental photoacoustic spectrum with previous measurements.

Crown Copyright @ 2007 Published by Elsevier B.V. All rights reserved.
\end{abstract}

Keywords: Fiber Bragg grating (FBG); Photoacoustic spectroscopy (PAS); Laser-induced opto-acoustic spectroscopy (LIOAS); Acousto-optic spectroscopy; Chemical sensing; $\mathrm{Er}_{2} \mathrm{O}_{3}$; Erbium oxide

\section{Introduction}

In recent years fiber Bragg gratings (FBGs) have undergone a spectacular transformation from passive optical reflectors to active devices [1-7] used, for example, in temperature [8], strain [9], chemical [10] and acoustic [11] measurements. FBGs have been developed primarily as "mirrors" for light which is transmitted through single mode fibers. In their simplest form they consist of a periodic modulation of refractive index in the core of the fiber. Light is reflected from these gratings at wavelengths for which the Bragg condition is met [2]. The use of non-uniform gratings, in which the local period or depth of refractive index modulation varies along the axis, endows the FBG with new properties, such as custom-shaped reflection

\footnotetext{
* Corresponding author. Tel.: +1 613533 2621; fax: +1 6135336669.

E-mail address: HPLoock@chem.queensu.ca (H.-P. Loock).
}

bands, an enlarged range of the reflection spectrum, or formation of more than one rejection band.

The emergence of modified FBGs and long-period gratings (LPGs) as chemical sensors is very promising, but has in the past been restricted to static measurements, i.e. those in which the response time is seconds or more. Here, we report on a new approach to chemical sensing based on time-resolved photoacoustic spectroscopy (PAS) [12-14]. With this technique the sensitivity of a FBG to strain is exploited as a means to detect the acoustic wave.

Because of its capability to quantify fast $(<\mu \mathrm{s})$ nonradiative processes time-resolved PAS is the technique of choice for numerous kinetic studies, especially in condensed matter and heterogeneous chemistry [14]. For example, time-resolved PAS was successfully used to evaluate directly the trap state energetics of photogenerated electrons in nanocrystalline colloidal titanium dioxide dispersed in aqueous solution [15]. Time-resolving such a 
process requires a transducer with a response of better than $1 \mu \mathrm{s}$ and in this case a $10 \mathrm{MHz}$ piezoelectric transducer was employed. The FBG has special potential in photoacoustic measurements on such solid samples since the photocatalyst can be supported by the fiber, which contains the FBG, thus opening a different avenue to measuring nonradiative processes. With this contribution we intend to illustrate the first use of FBGs as fast, "all-optical" detectors for photoacoustic waves.

It is well known that FBGs can be used as "microphones", and there exists a considerable body of work describing the use of FBGs particularly as ultrasound transducers [11,16-26]. Furthermore, the capability of FBGs to detect acoustic waves in real-time makes them well suited in measuring nonradiative emission processes which are commonly initiated by photoexcitation of solids and liquids. An acoustic wave traveling through a supported FBG initiates a spectral change, similar to those initiated by a source of strain [27-32]. We found that the FBGs employed in the present study have the ability to respond to $>7 \mathrm{MHz}$ photoacoustic waves generated by photoexcitation of a solid sample with a nanosecond laser pulse. As will be shown below, the response time is limited in our case by the instrumental configuration used to interrogate the FBG and not inherently by the FBG itself.

To demonstrate the capability of the FBG "microphone" as a photoacoustic sensor, an acoustic wave was generated by $<10 \mathrm{~ns}$ pulsed excitation of erbium oxide powders sandwiched between a thin glass slide and quartz plate. Erbium oxide is widely used as a dopant for optical fibers which are used in fiber lasers and optical amplifiers. The excitation was to a resonant excited state at around $520 \mathrm{~nm}$ and the photoacoustic response was recorded in real-time by detecting either the reflection or the transmission of the clamped FBG. The experimental time-resolved photoacoustic FBG spectrum is consistent with the calculated and experimental response assuming a local transverse load of a few Newtons. The focus of this report is the description of the operating principle of a new transducer and its characterization. A detailed comparison to existing photoacoustic techniques, in particular with respect to sensitivity and time resolution, is beyond the scope of this work and will be reported in the near future.

\section{Experimental details}

The setup of the FBG photoacoustic sensor is shown in Fig. 1. A continuous wave (cw) tunable laser diode (Ando AQ4320D; $\lambda=1520 \mathrm{~nm}-1620 \mathrm{~nm} ; \Delta \lambda=1.6 \mathrm{pm}$ ) was used to probe the FBG response either by measuring its reflection or its transmission. The wavelength of the laser was selected so that it coincided with one of the reflection edges of the FBG. The FBGs were made by ITF Labs (Montréal, QC, Canada) had a period of $530 \mathrm{~nm}$, a length of $7 \mathrm{~mm}$ and a maximum attenuation of $16 \mathrm{~dB}$ at around $1550 \mu \mathrm{m}$. Other FBGs with different period and attenuation spectra were also used and, as expected, their sensitivity to the

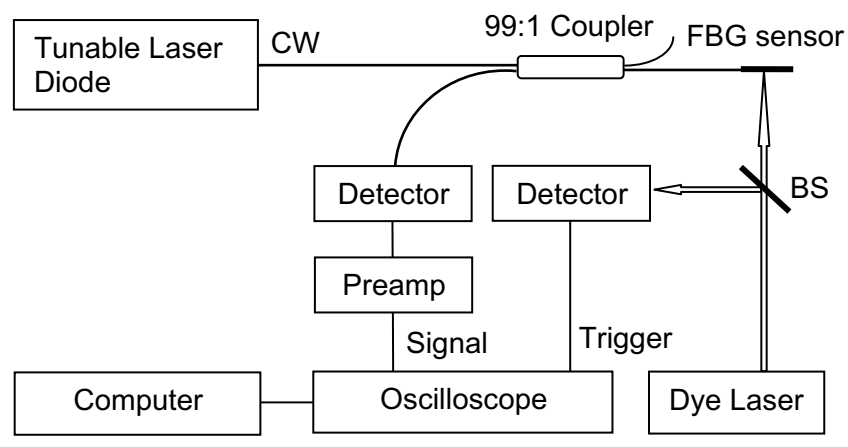

Fig. 1. Experimental setup for detecting time-resolved photoacoustic signals using a fiber Bragg grating sensor. Shown is the setup used for measuring the FBG reflectance spectrum.

photoacoustic wave increased with the slope of the spectrum, $\mathrm{d} T / \mathrm{d} \lambda$, at the probe laser wavelength. The laser was directed into the fiber Bragg grating using a coupler (Lightel Technology, splitting ratio 1:99, Corning SMF28). The reflected light was directed to a detector (Thorlabs, D400FC, InGaAs, rise time: $0.1 \mathrm{~ns}$ ), and the signal was amplified (Panametrics, $10 \mathrm{MHz}$, Tech Inc. USA) and averaged by an oscilloscope (Tektronix, TDS3054B, $500 \mathrm{MHz}, 5 \mathrm{GS} / \mathrm{s}$ ), before being transferred to a computer.

The time-resolved reflection or transmission spectra were recorded using two configurations shown in Fig. 2a and $b$. In these configurations two different samples were used. Carbon black is known to have very fast and strong photoacoustic response due to a rapid radiationless decay of the primary excited state $(\tau<10 \mathrm{~ns})$. For the initial characterization carbon black was therefore used as a sample in Configuration $\mathrm{A}$ and excited by a pulsed $\mathrm{Nd}$ :YAG laser (third harmonic at $355 \mathrm{~nm}, 10 \mathrm{~mJ} /$ pulse, $\Delta t<10 \mathrm{~ns}$, irradiated area is $\sim 1 \mathrm{~mm}^{2}$ ). Carbon black was also used as a time-reference in Configuration B. Additionally, $\mathrm{Er}_{2} \mathrm{O}_{3}$ was used as a solid sample in both Configurations A and B.
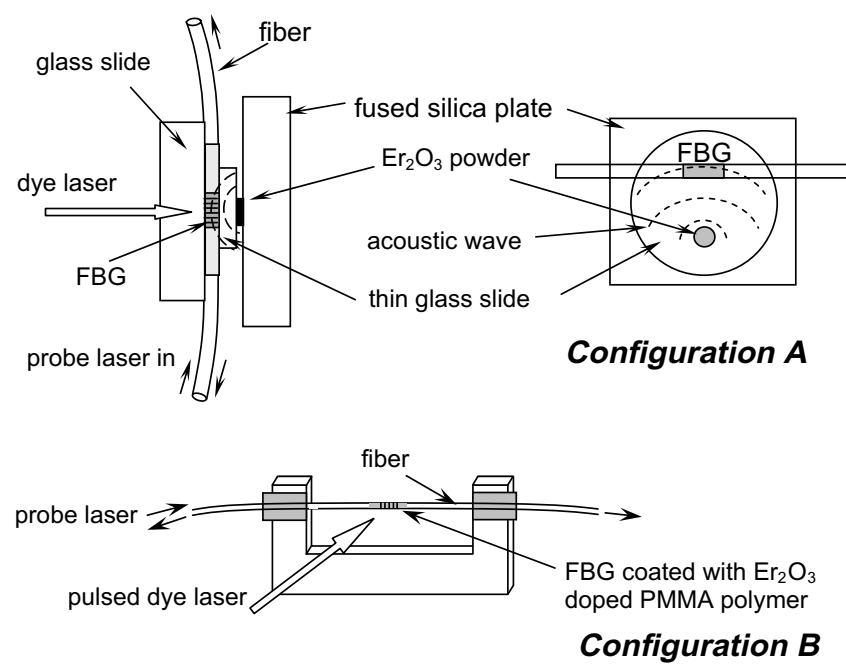

Fig. 2. Schematic illustration of the two fiber Bragg grating configurations. FBGs in both configurations were interrogated in reflection and transmission mode. 
$\mathrm{Er}_{2} \mathrm{O}_{3}$ has an absorption spectrum with sharp, distinct features at around $520 \mathrm{~nm}$ and is used here to compare our method with previously recorded spectra. In Configuration A, a suspension of the erbium oxide powder (Sigma-Aldrich, 99.99\% purity) in water was sandwiched between a round thin glass slide (diameter of $15 \mathrm{~mm}$, and thickness of $0.146 \mathrm{~mm}$ ) and a fused quartz slide ( $1.5 \mathrm{~mm}$ thick) and the liquid was allowed to evaporate. The thickness of the solid sample layer is then about $50 \mu \mathrm{m}$. Another glass slide (1.5 mm thick) was used to clamp the fiber Bragg grating onto the surface of the thin glass slide. In this configuration the excitation pulse propagated sequentially through the thick glass slide, passes the FBG, propagates through the thin slide, and then the sample, striking the sample only $146 \mu \mathrm{m}$ from the FBG. The photoacoustic response from the slides and the FBG alone, i.e. without a sample, was not detectable. The pulsed dye laser output was gently focused on the sample (Lambda Physik, Scanmate 2E, irradiated area approx. $1 \mathrm{~mm}^{2}, 120$ to $450 \mu \mathrm{J} /$ pulse, $\Delta t=10 \mathrm{~ns}$, band width $0.2 \mathrm{~cm}^{-1}$, at $10 \mathrm{~Hz}$ repetition).

When using Configuration B, a solid thin film sample was prepared by co-doping $\mathrm{Er}_{2} \mathrm{O}_{3}(15 \%$ by weight $)$ and carbon black $(2 \%)$ in a polymer matrix of a polymethylmethacrylate (PMMA) host. The film was prepared by suspending $\mathrm{Er}_{2} \mathrm{O}_{3}$ and carbon black in a solution of PMMA in acetone. The viscous suspension was then used to coat either a cleaned glass slide or the surface of the fiber Bragg grating. The polymer solidified upon evaporation of the solvent. To obtain an estimate for the time-constant of the radiationless decay of $\mathrm{Er}_{2} \mathrm{O}_{3}$ the photoacoustic response was obtained by excitation of the sample on seven of the strongest resonance features between 519 and $527 \mathrm{~nm}$ as well as seven other wavelengths. To obtain the time-constants for the heat release function, a deconvolution with a reference waveform (recorded off-resonance) is necessary. Care was taken that the excitation position remained unchanged while sampling the 14 waveforms.

To obtain the photoacoustic spectrum of $\mathrm{Er}_{2} \mathrm{O}_{3}$ the dye laser excitation wavelength was tuned between $519 \mathrm{~nm}$ and $527 \mathrm{~nm}$ either in $100 \mathrm{pm}$ steps with 512 averages per wavelength, or $5 \mathrm{pm}$ steps with 32 averages per laser wavelength and subsequent 10 point averaging. For each laser shot the change of the reflectivity of the FBG at $1549.73 \mathrm{~nm}$ was recorded for $15 \mu$ s with up to $4 \mathrm{~ns}$ temporal resolution.

The static transmission spectrum of the FBG under lateral local pressure was determined using the setup sketched in Fig. 3. The center region of the FBG was stressed using an incremental load applied by a glass rod, and the transmission spectrum was recorded using an optical spectrum analyser (Agilent 8614).

\section{Results and discussion}

$\mathrm{Er}_{2} \mathrm{O}_{3}$ shows a number of narrow absorption features at around $520 \mathrm{~nm}$. Using Configuration $\mathrm{A}$ and laser excitation at 81 wavelengths between $519 \mathrm{~nm}$ and $527 \mathrm{~nm}$ the

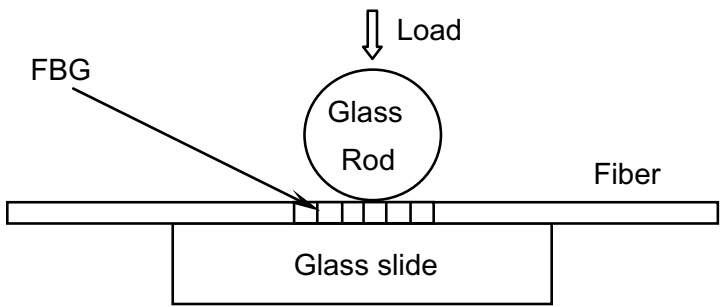

Fig. 3. Illustration of the transverse load sensing experiment. The center of a FBG is stressed using a variable load applied to a glass rod with $4 \mathrm{~mm}$ diameter.
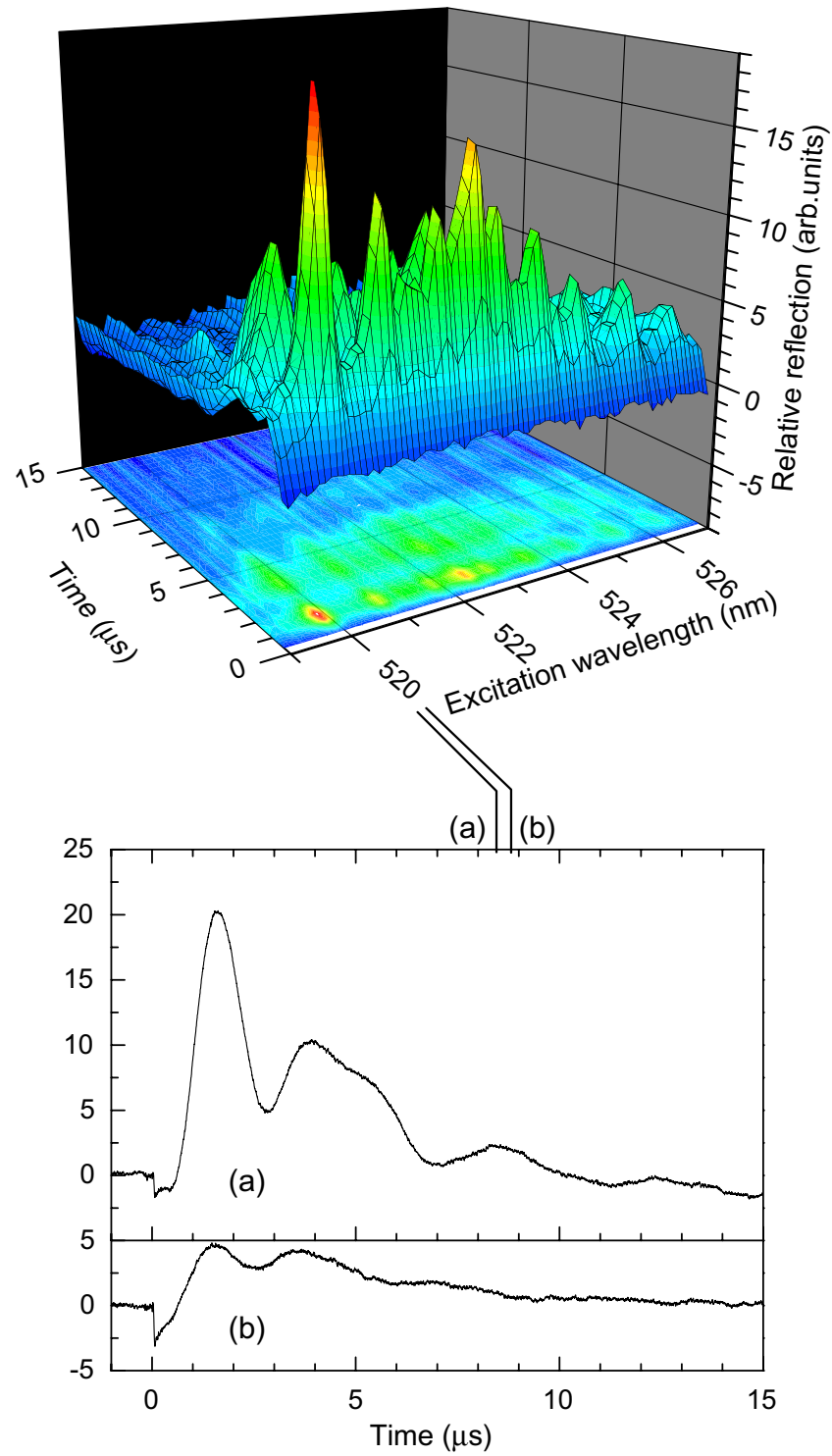

Fig. 4. Composite plot of 81 photoacoustic response curves obtained by excitation of $\mathrm{Er}_{2} \mathrm{O}_{3}$ between $519 \mathrm{~nm}$ and $527 \mathrm{~nm}$. The FBG in Configuration $\mathrm{A}$ was interrogated with the probe laser tuned to a wavelength which corresponds to the absolute minimum in Fig. 6. Two of the 81 timeresolved reflectivity spectra are shown in lower graph. The photoacoustic response is larger when tuning the excitation wavelength to an absorption feature at $519.9 \mathrm{~nm}$ (a), compared to off-resonance excitation at $520.3 \mathrm{~nm}$ (b). Also apparent are 3-4 $\mu$ s echoes of the intense initial signal from the interface of the glass plate assembly. The displayed signal was corrected for laser power fluctuations. 
reflection of the FBG was recorded for $15 \mu \mathrm{s}$ at $1549.73 \mathrm{~nm}$. A composite 3D plot is presented in Fig. 4. Two time-resolved waveforms recorded on-resonance and off-resonance with $\mathrm{Er}_{2} \mathrm{O}_{3}$ transitions are included.

The spectrum in Fig. $5 \mathrm{~b}$ represents a slice of Fig. 4, in which the transmission was measured $1.5 \mu$ s after laser excitation. Similarly, Fig. 5c was obtained by measuring the transmission of the FBG in Configuration B $600 \mathrm{~ns}$ after excitation. To compare with previously published photoacoustic spectra it is assumed that the change in the FBG's reflectivity is proportional to the sample's absorption.

The peak positions and intensities of the photoacoustic spectrum shown in Fig. 5 compare well with a previously reported photoacoustic spectrum shown in Fig. 5a [33], which was recorded using lower time resolution. This spectrum was recorded using a conventional piezoelectric microphone, a $1 \mu$ s laser pulse, and a $4 \mu$ s integration of the photoacoustic response waveform. The similarity of Fig. 5a-c confirms that the change in the FBG's reflectivity is indeed proportional to the $\mathrm{Er}_{2} \mathrm{O}_{3}$ absorption.

The relative intensities in Fig. $5 \mathrm{~b}$ and c deviate slightly from previous data, however, and we have presently no

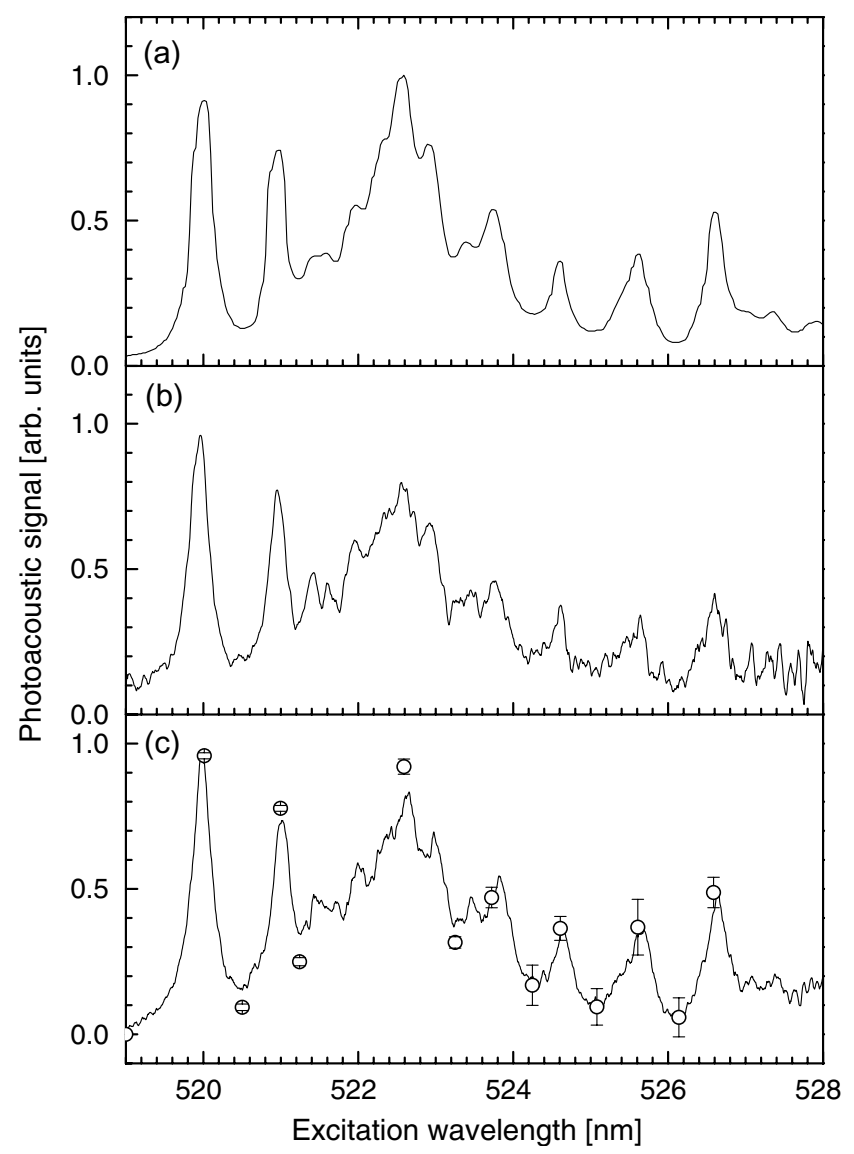

Fig. 5. The photoacoustic spectrum of $\mathrm{Er}_{2} \mathrm{O}_{3}$ (a) adapted from Ref. [33] (b) recorded using Configuration $\mathrm{A}$ and (c), using Configuration $\mathrm{B}$. The circles in panel (c) are the ratios of the integrated intensities of the slow to the fast decay process, which were obtained from the deconvolution analysis shown in Fig. 8. explanation for the differences. As reasons for the intensity differences we exclude depletion of the sample and bulk heating effects by noticing that spectra recorded consecutively are very similar. The signal was corrected for excitation laser power assuming a linear relationship between laser energy and photoacoustic signal. Due to the lower conversion efficiency of the laser dye, the excitation laser power decreased when tuning to longer wavelengths. By correcting for this laser power decrease, we also increase the noise with increasing wavelength (Fig. $5 \mathrm{~b}$ and c). Clearly the noise in the spectrum is largely due to fluctuations in the excitation laser power and to a much lesser extent to mechanical distortions of the FBG. The drift of the probe laser wavelength is specified as $<0.8 \mathrm{pm} / \mathrm{h}$ and is expected to be negligible. It is apparent from Fig. 5 that, despite the laser power fluctuations, one can obtain a quantitatively accurate signal by integrating as little as $100 \mathrm{~ns}$ of the FBG response. It is expected that the noise due to fluctuation in excitation laser intensity can be greatly reduced simply by coupling the device to a laser with a stabilized output and longer averaging at a higher repetition rate.

Ideally, the PAS response shall be linear over a large range of photoacoustic powers. The linear range is strongly dependent on the probe wavelength that is selected for a given FBG (see below). The dynamic range of our measurement was determined by recording the amplitude of the transmitted signal for different mechanical loads (see Section 3.5). The absorbance of the grating signal was found to be linear up to a load of $400 \mathrm{mN}$. This corresponds to a range of absolute source acoustic powers between $95 \mathrm{~dB}$ and over $110 \mathrm{~dB}$.

\subsection{Sensitivity to probe wavelength}

In order to characterize the FBG-PAS setup and to determine the wavelength of the probe laser that gives the most accurate response, a dynamic transmission spectrum was recorded using carbon black as a sample and using pulsed Nd:YAG laser excitation at $355 \mathrm{~nm}$ in Configuration A. Fig. 6 shows that $\sim 600 \mathrm{~ns}$ after the excitation laser pulse the transmission spectrum of the FBG is distorted by the acoustic wave and that the originally symmetric transmission spectrum has split into a portion that shifts to longer wavelength and another one that shifts to shorter wavelength. By subtracting the original FBG spectrum we obtained a spectral response pattern that is characteristic for birefringence induced by a lateral load. As will be shown below one can understand that the distortion of the FBG arises from the non-uniform pressure caused by the acoustic wave. When loaded laterally, the transmission spectrum will be influenced by the mechanically induced birefringence and the two polarization states will shift in opposite directions. It is noteworthy that the intensity of the distortion is time-dependent (Fig. 7), i.e. that the distortion spectrum changes only in amplitude, but that the maxima and minima remain at the same wavelengths. 


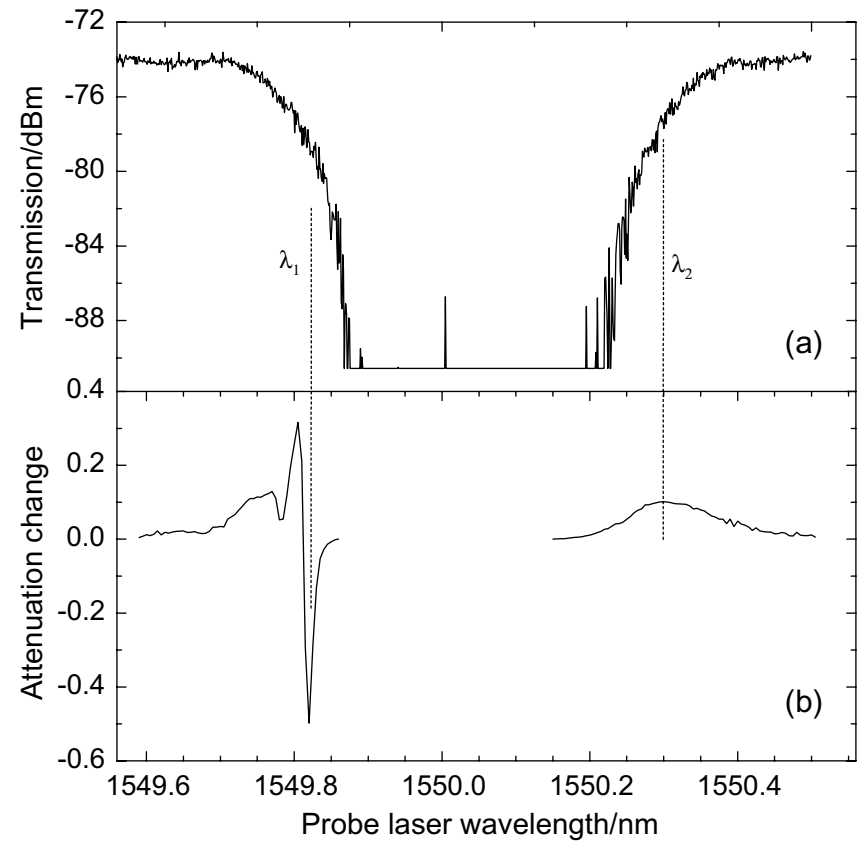

Fig. 6. The static FBG transmission spectrum (a), and the differential transmission spectrum (b). Both were recorded by photoexcitation of carbon black at $355 \mathrm{~nm}$ using Configuration A. The differential transmission spectrum was obtained from the temporal response curves of Fig. 7 by subtracting the static transmission from the transmission at $600 \mathrm{~ns}$. The probe wavelength interval is $5 \mathrm{pm}$. Both extrema at $\lambda_{1}=1549.82 \mathrm{~nm}$ and at $\lambda_{2}=1550.300 \mathrm{~nm}$ can be used to record PAS. Note that the characteristic oscillation at short wavelengths is characteristic for birefringence.
Using the information from Fig. 6 we can maximize the sensitivity by setting the probe laser wavelength to $\lambda_{1}=1549.830 \mathrm{~nm}$ and fixing the readout delay to $\sim 600$ ns. The echoes apparent in Fig. 7 have a period of $840 \mathrm{~ns}$ and are due to reflections on the surfaces of the quartz disks of Configuration $\mathrm{A}$ in Fig. 2 (characteristic dimension: about $3.3 \mathrm{~mm}$ ).

We note that in our experiment the amplitude of the time-resolved PAS signals strongly depends on the excitation position on the surface of the sample layer. Not surprisingly, by choosing an excitation position close to the center of the FBG the signal is maximized and interferences from echoes are reduced. Presently, no attempt was made to correlate the signal's intensity to the heat release of the photoacoustic process. Similarly, the quantification of the nonradiative transition probability and of the heat release kinetics will be the subject of future studies.

\subsection{Deconvolution analysis}

A time-resolved photoacoustic response obtained similarly to those shown in Fig. 4 was deconvoluted to determine the validity of the static model described below and to estimate the uncertainty in the decay constants that are obtained using the FBG-PAS technique. The observed signal, $S(t)$, is the convolution of the heat source function, $H(t)$, with the instrument response function, $R(t)$ $[34,35]$.

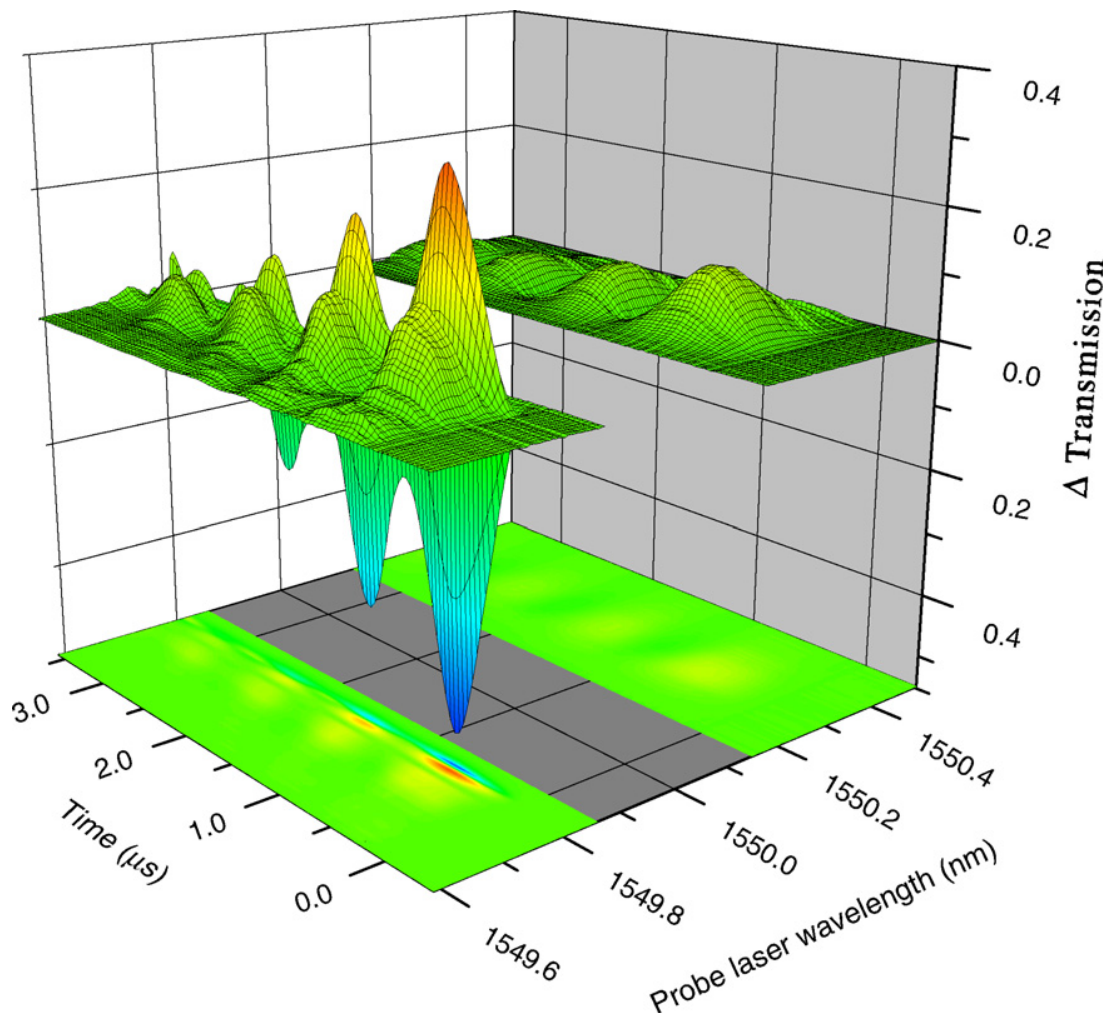

Fig. 7. Time dependence of the differential FBG transmission spectrum as shown for one delay in Fig. 6b. The graph shows that the distortion of the transmission spectrum changes in amplitude but not in shape as the acoustic wave travels through the FBG. The time resolution is 20 ns and the probe laser wavelength interval is $5 \mathrm{pm}$. 
$S(t)=\int R\left(t-t^{\prime}\right) H\left(t^{\prime}\right) \mathrm{d} t^{\prime}$

The photoacoustic response was obtained using Configuration $\mathrm{B}$ by photoexcitation of a thin film of PMMA polymer co-doped with $\mathrm{Er}_{2} \mathrm{O}_{3}$ and carbon black on seven of the strongest resonance features of the spectrum as well as seven other wavelengths. The time-constants were obtained by deconvolution of the response waveform with a waveform recorded off-resonance at $519 \mathrm{~nm}$. At this wavelength carbon black is the only source of the photoacoustic signal, since absorption of the PMMA and the fiber was found to be negligible. At all other wavelengths the time-resolved photoacoustic response will show contributions from, both, carbon black and $\mathrm{Er}_{2} \mathrm{O}_{3}$ and, consequently, the heat source function is expected to be a biexponential decay with lifetimes $\tau_{1}$ and $\tau_{2}$. The ratio of the time-integrated photoacoustic signal originating from $\mathrm{Er}_{2} \mathrm{O}_{3}$ to the signal originating from carbon black will be wavelength dependent and be proportional to the absorbance of $\mathrm{Er}_{2} \mathrm{O}_{3}-$ assuming, of course, that the absorption cross section of carbon black is constant in the 519-527 nm spectral range.

The deconvolution was carried out using commercial software (Sound Analysis Software Version 1.50 D, Quantum Northwest). For the wavelengths on-resonance with $\mathrm{Er}_{2} \mathrm{O}_{3}$ spectral features, the signal could readily be deconvoluted by the reference waveform and a transfer function, which is a biexponential decay function. The deconvolution software gave exponential decay constants of which one was found to be less than $\tau_{1}=1 \mathrm{~ns}$ and one was about $1 \mu \mathrm{s}$. Our assumption that these decay processes correspond to the temporal photoacoustic response of carbon black and $\mathrm{Er}_{2} \mathrm{O}_{3}$, is consistent with the fact that the ratio of the two time-integrated contributions follows the intensity of the pure $\mathrm{Er}_{2} \mathrm{O}_{3}$ spectrum. (see Fig. 5c). For the wavelengths recorded off-resonance, $\tau_{1}$ was fixed at $1 \mathrm{~ns}$ and the ratio between the contributions and $\tau_{2}$ were obtained by deconvolution.

The lifetime of $\mathrm{Er}_{2} \mathrm{O}_{3}$ was determined to be $\tau_{2}=$ $1060 \mathrm{~ns} \pm 140 \mathrm{~ns}$, where the uncertainty in this measurement was estimated by forward convolution of the reference signal with biexponential decays with lifetime $\tau_{1}=$ $1 \mathrm{~ns}$ and the intensity ratio fixed at the value given by the spectrum in Fig. 5c. The criterion used to specify the uncertainty in the measurement, is given by the increase in the root-mean square difference between this generated waveform and the photoacoustic signal by a factor of three (Fig. 8).

\subsection{Time resolution}

Fomitchov showed that $0.5-5.0 \mathrm{MHz}$ ultrasound can be detected by a FBG [36]. The time resolution of a FBG photoacoustic system is ultimately limited by the dimensions of the FBG sensor, here of length $7 \mathrm{~mm}$, and core diameter of $8.2 \mu \mathrm{m}$. The transit time of an infinitely short, plane acoustic wave traveling through the FBG can be esti-

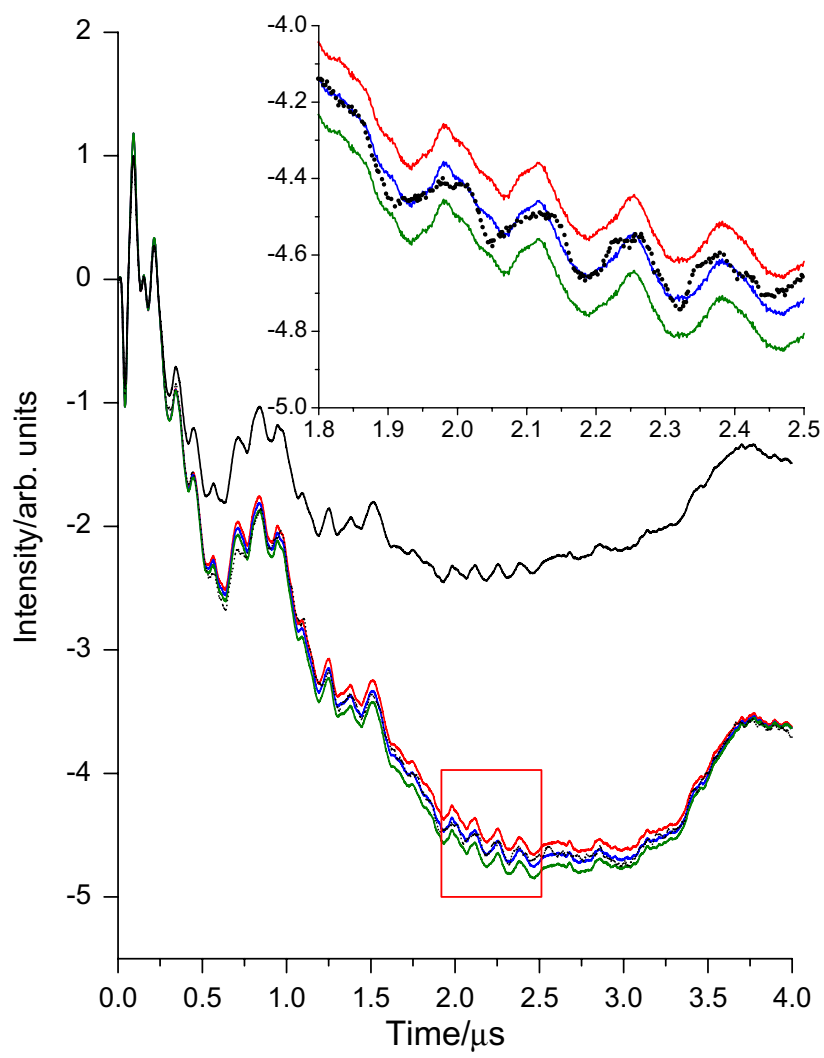

Fig. 8. Deconvolution analysis of the photoacoustic response obtained using Configuration B. A film of PMMA was doped with carbon black and $\mathrm{Er}_{2} \mathrm{O}_{3}$. The reference (top black curve) was obtained from offresonance excitation at $519 \mathrm{~nm}$ (photoacoustic response from carbon black only) while the signal was obtained by excitation at $520.012 \mathrm{~nm}$ (lower black curve). The simulated curves show a convolution of the reference signal with biexponential decays. One of the lifetimes was set to $1 \mathrm{~ns}$ and the other to $1200 \mathrm{~ns}, 1061 \mathrm{~ns}$ and $930 \mathrm{~ns}$, respectively (red curves from top) (For interpretation of the references to color in this figure legend, the reader is referred to the web version of this article).

mated by the speed of sound in quartz, about $3630 \mathrm{~m} / \mathrm{s}$ [36] and the FBG's dimensions. For an acoustic wave traveling along the fiber, the response time is limited to about $2 \mu$ s. On the other hand, any photoacoustic source far above the FBG produces a nearly planar acoustic wave traveling across the FBG and can initiate a response that may be as short as the transit time through the fiber core - here, 2 ns. Clearly, this limit was not reached in these experiments but temporal features as brief as $50-100 \mathrm{~ns}$ are reliably reproduced (Fig. 8). As was shown above, a deconvolution analysis can provide for the measurement of decay constants with an uncertainty of less than $150 \mathrm{~ns}$.

In both Configurations A and B the time resolution is limited by the presence of scattered acoustic waves and echoes and not by the interaction time of the FBG with the acoustic wave. Improvements may be possible by moving the FBG further away from the photoacoustic point source so that a smaller solid angle of the spherical wave is sampled. Also, larger dimensions of the supporting plates will help avoid scattering of the acoustic wave before it reaches the FBG. 


\subsection{Theoretical analysis assuming a static model}

The interaction time of the photoacoustic wave with the FBG is one or two orders of magnitude longer than the time resolution of the spectroscopic measurement $(\delta t=3 \mathrm{~ns}$ ), so that the passage of the photoacoustic wave through the FBG can be treated as a slowly evolving system. In the analysis detailed below we determine the spectrum of the FBG at a fixed time after the photoacoustic pulse was initiated. The model has the advantage that it can be verified in two independent ways, first by recording the FBG spectrum at the time of maximum distortion (about 600 ns in Figs. 4 and 8) and, secondly, by simply straining the FBG with a load that exerts a strain equivalent to the acoustic wave (Fig. 3).

In what follows it is assumed that the FBG is an apodized fiber Bragg grating with a refractive index modulation depth profile described by

$\delta n_{\text {eff }}(z)=\delta n_{\max } \exp \left[-\tau\left(\frac{z-L / 2}{L}\right)^{2}\right]$

where $\delta n_{\max }=5.8 \times 10^{-4}$ is the maximum of the refractive index modulation depth, $L=7 \mathrm{~mm}$ is the length of FBG, and $\tau=10$ is the attenuation constant. The lateral static force acts on only a small section - about one tenth of the length of FBG - near the center of the FBG. The stress induced changes of the effective refractive index, $\Delta n_{\text {eff }}=n_{\text {eff }}^{0}-n_{\text {eff }}$, can be calculated using photoelasticity theory $[27,28]$

$$
\begin{aligned}
\left(\Delta n_{\mathrm{eff}}\right)_{x}= & -\frac{n_{0}^{3}}{2 E}\left\{\left(p_{11}-2 v p_{12}\right) \sigma_{x}\right. \\
& \left.+\left[(1-v) p_{12}-v p_{11}\right]\left(\sigma_{y}+\sigma_{z}\right)\right\} \\
\left(\Delta n_{\mathrm{eff}}\right)_{y}= & -\frac{n_{0}^{3}}{2 E}\left\{\left(p_{11}-2 v p_{12}\right) \sigma_{y}\right. \\
& \left.+\left[(1-v) p_{12}-v p_{11}\right]\left(\sigma_{x}+\sigma_{z}\right)\right\}
\end{aligned}
$$

with

$$
\begin{aligned}
\sigma_{x} & =\frac{2 F}{\pi L_{\mathrm{eff}} D} \\
\sigma_{y} & =-\frac{6 F}{\pi L_{\mathrm{eff}} D} \\
\sigma_{z} & =v\left(\sigma_{x}+\sigma_{y}\right)
\end{aligned}
$$

where for silica Young's modulus, $E=74.52 \mathrm{GPa}$, and Poisson's coefficient, $v=0.17$. The strain-optic coefficients $p_{11}=0.121$ and $p_{12}=0.270$ were obtained from Ref. [28]. $\sigma_{x}$ and $\sigma_{y}$ are the stress components of the grating, $F$ is the applied force on the fiber, and $D=125 \mu \mathrm{m}$ is the diameter of the SMF-28 fiber. The effective interaction length $L_{\text {eff }}$ is estimated at $1 \mathrm{~mm}$.

Based on the piecewise-uniform approach (transfer matrix formalism) [37], the grating is divided into $N$ uniform subgratings - in our model, $N=200$. We define $a^{+}$ and $a^{-}$as the slowly varying amplitudes of the forward and backward traveling waves, respectively. The propaga- tion through each uniform section for each polarization state is described by a matrix defined as:

$$
\left[\begin{array}{c}
a_{j}^{+} \\
a_{j}^{-}
\end{array}\right]=T^{(j)} \cdot\left[\begin{array}{c}
a_{j+1}^{+} \\
a_{j+1}^{-}
\end{array}\right]=\left[\begin{array}{ll}
T_{11}^{(j)} & T_{12}^{(j)} \\
T_{21}^{(j)} & T_{22}^{(j)}
\end{array}\right] \cdot\left[\begin{array}{c}
a_{j+1}^{+} \\
a_{j+1}^{-}
\end{array}\right]
$$

where

$$
\begin{aligned}
& T_{11}^{(j)}=T_{22}^{(j) *}=\cosh \left(\gamma \Delta z_{j}\right)-\mathrm{i} \frac{\Delta \beta}{\gamma} \sinh \left(\gamma \Delta z_{j}\right) \\
& T_{12}^{(j)}=T_{21}^{(j) *}=-\mathrm{i} \frac{\kappa}{\gamma} \sinh \left(\gamma \Delta z_{j}\right) \\
& \Delta \beta=\beta-\beta_{0}=2 \pi n_{\text {eff }}\left(\frac{1}{\lambda}-\frac{1}{\lambda_{\mathrm{B}}}\right) \\
& \lambda_{\mathrm{B}}=2 n_{\text {eff }} \Lambda \\
& \kappa=\frac{\pi}{\lambda} \delta n_{\text {eff }} \\
& \gamma=\sqrt{\kappa^{2}-\Delta \beta^{2}}
\end{aligned}
$$

Here, $\Lambda$ is the grating constant of $530.057 \mathrm{~nm}, \beta=\pi / \Lambda$, is the light wave propagation constant, $\Delta \beta$ is the differential propagation constant, the effective refractive index of the FBG core mode $n_{\mathrm{eff}}^{0}=1.46212$, and therefore the propagation for each polarization state through the whole grating is given by:

$\left[\begin{array}{c}a_{0}^{+} \\ a_{0}^{-}\end{array}\right]=T^{(1)} T^{(2)} \ldots T^{(j)} \ldots T^{(N)} \cdot\left[\begin{array}{c}a_{N}^{+} \\ a_{N}^{-}\end{array}\right]$

Considering the boundary condition

$\left[\begin{array}{c}a_{N}^{+} \\ a_{N}^{-}\end{array}\right]=\left[\begin{array}{l}1 \\ 0\end{array}\right]$

one can calculate the reflectivity, $R$, as

$R=\left|\frac{a_{0}^{-}}{a_{0}^{+}}\right|^{2}$

Since, the resulting reflection or transmission spectrum is different for the parallel and perpendicular polarization components, this spectrum will depend on the initial polarization ratio - here assumed to be $1: 1$. The calculation yields spectra of the FBG (Fig. 9) that are in very good agreement to those observed using a static load and to those obtained by recording a dynamic spectrum $\sim 600$ ns after laser pulse excitation of the photoacoustic device (Fig. 6). A similar oscillation in the differential spectrum is observed at the short wavelength edge of the FBG, while a more uniform intensity increase at the long wavelength side is found. This confirms that the dynamic response of the FBG spectrum to a spherical acoustic wave, which has its origin above the FBG, can be understood as being caused by a local and time-dependent source of lateral strain.

Of course, this model will not apply if the acoustic wave travels along the fiber as it does for echoes from the mounting bracket in Configuration B (not shown in Fig. 8). Echoes were observed every $22.7 \mu$ s after initial excitation - consistent with a distance of $77 \mathrm{~mm}$ between the brackets (not 


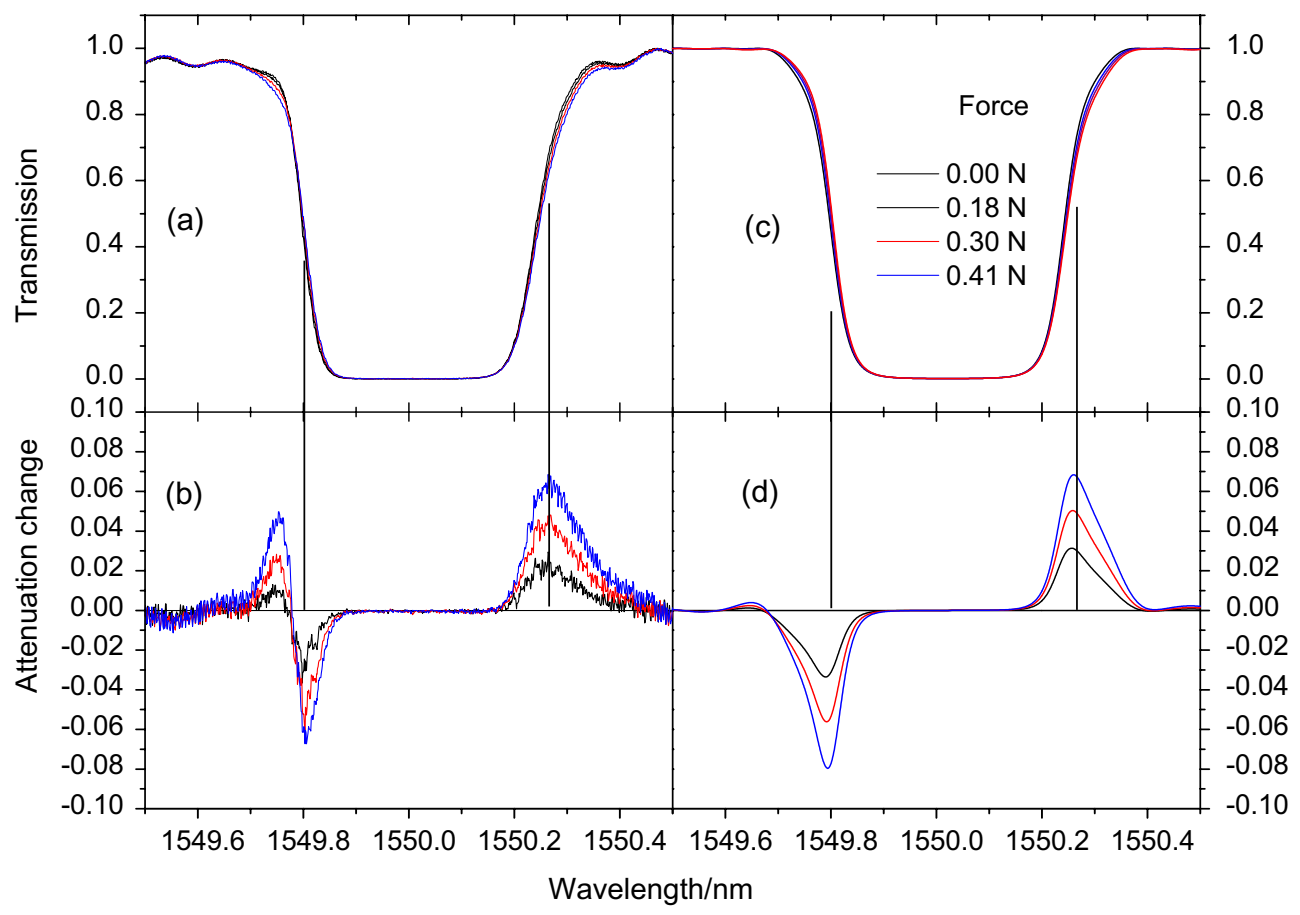

Fig. 9. Distortion of the static transmission spectrum caused by applying stress to the center of the FBG (see Fig. 3). Panel (a) shows the raw transmission spectrum and Panel (b) shows the difference between the stressed and unstressed FBG. The respective calculated spectra using the static model are shown in panels (c) and (d).

shown in Fig. 8). These echoes affect the grating period and thereby shift the spectrum, but they do not induce the birefringence which is observed when the source of photoacoustic excitation lies on the surface of the grating.

\subsection{Sensitivity and detection limit}

It is possible to estimate the absolute sensitivity and minimal detectable acoustic power of our device by determining the increase in the FBG's attenuation with increased static load (Figs. 10 and 6). The transmission follows a linear trend up to over $400 \mathrm{mN}$ (Fig. 10) and the sensitivity of the device is then trivially given by the slopes as $\mathrm{d} T / \mathrm{d} F=1.64 \mathrm{~dB} / \mathrm{N}$ at $1550.264 \mathrm{~nm}$ or $-1.38 \mathrm{~dB} / \mathrm{N}$ at $1549.803 \mathrm{~nm}$.

The uncertainty of the linear fit gives an upper limit to the minimal detectable transmission change due to a static force as $0.02 \mathrm{~dB}$ (corresponding to a force of $12 \mathrm{mN}$ ). This limit is determined by the measurement precision of the optical spectrum analyzer $(0.1 \mathrm{~dB})$ and the accuracy with which the load can be adjusted.

Similarly, the minimal detectable transmission change in the acoustic measurements of Fig. 6 may be estimated from the noise level to be below $3 \times 0.03 \mathrm{~dB}=0.09 \mathrm{~dB}$, corresponding to a force of $F=60 \mathrm{mN}$. This high estimate to the detection limit is indicative of our light detection configuration and lower detection limits can be realized e.g. by lock-in detection of the reflected light or use of more sensitive light detectors.

To relate the applied force to source sound power, one may use the relation

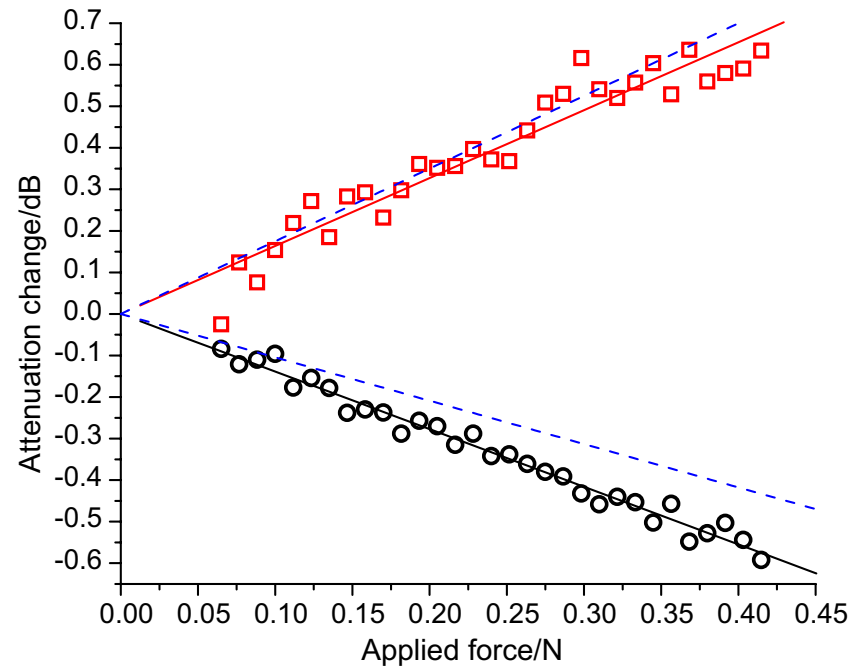

Fig. 10. Attenuation change of the FBG at $1550.264 \mathrm{~nm}$ (red squares) and at $1549.803 \mathrm{~nm}$ (black circles) as a function of the lateral static load (see Fig. 3). The linear fits (solid lines) give sensitivities of $1.68 \mathrm{~dB} / \mathrm{N}$ and $-1.38 \mathrm{~dB} / \mathrm{N}$, respectively. The dashed lines are obtained from the model and correspond to sensitivities of $+1.75 \mathrm{~dB} / \mathrm{N}$ and $-1.04 \mathrm{~dB} / \mathrm{N}$ (For interpretation of the references to color in this figure legend, the reader is referred to the web version of this article).

$P_{\mathrm{ac}}=\frac{F^{2}}{A c \rho}=0.95 \frac{W}{N^{2}} F^{2}$

where the area over which the pressure is applied corresponds to the (lengthwise) cross sectional area of the fiber $A=1 \mathrm{~mm} \times 0.125 \mathrm{~mm}=1.25 \times 10^{-7} \mathrm{~m}^{2}$, the speed of 
sound is about $3500 \mathrm{~m} / \mathrm{s}$ and the density of the material is estimated as $2400 \mathrm{~kg} / \mathrm{m}^{3}$.

For an acoustic force acting normal to the FBG it can be assumed that the relevant area, $A$, of the FBG that responds to the sound wave is the same as in the static experiment. The minimal detectable sound power can then be estimated simply as $P_{\mathrm{ac}}=0.95 \mathrm{~W} / \mathrm{N}^{2} \times(60 \mathrm{mN})^{2}=$ $3.4 \mathrm{~mW}$. To relate this to a commonly used scale, one may use the auditory threshold $\left(10^{-12} \mathrm{~W}\right)$ as a reference to determine the acoustic power in decibels - here, $L_{\mathrm{ac}}=10 \log \left(P_{\mathrm{ac}} / 10^{-12} \mathrm{~W}\right)=95 \mathrm{~dB}$. This detection threshold - consistent with the lack of FBG response to ambient noise - is roughly 7 orders of magnitude less compared to commercial microphones, which achieve typically a detection threshold of $\angle 25 \mathrm{~dB}$. If necessary, the sensitivity and detection limit can be improved by using more sensitive light detectors, lock-in detection or cavity-enhanced detection. In the latter case the FBG would be one of the two reflectors needed to create a high finesse optical cavity.

\section{Conclusion}

In time-resolved photoacoustic spectroscopy a suitable microphone is characterized by high frequency response, ruggedness and sensitivity. Considering these requirements, fiber Bragg gratings make good acoustic transducers and have the added advantage of being insensitive to electrical interferences and comparably resistant to corrosive environments.

As we demonstrated a time response to an acoustic wave with about $150 \mathrm{~ns}$ resolution is readily achieved and could be further improved using a different configuration. The temporal evolution of the FBG's spectra in response to an acoustic wave can be fully modeled assuming that the acoustic wave induces birefringence in the FBG similar to what was observed using a static transverse load.

Erbium oxide powders were used as test samples because they have an intense structured absorption in the visible region of the spectrum. We believe that the technique is equally suitable for time-resolved PAS measurements of other condensed samples including corrosives which may be difficult to interrogate using conventional microphones.

The small dimensions of this photoacoustic system are particularly attractive for sensitive measurements of radiationless energy transfers. Photoacoustic spectroscopy is likely unique in having the highest sensitivity when the interaction volumes and detection systems are smallest. Because of their micron-sized dimensions optical waveguides provide opportunities to shrink the detection region to dimensions that are less than millimeters.

In an ideal PAS setup the time resolution is limited by the laser pulse width and not by the detection system. In our experiment the time resolution is presently limited by the response of the FBG detector assembly and also by scattered acoustic waves that overlap with the initial acoustic wave at short times. Nevertheless, it was shown that the
FBG has a good temporal response and can readily resolve features that are shorter than $100 \mathrm{~ns}$.

If time resolution is not required, excitation with a modulated light source operating at the FBG assembly's resonant frequency is advisable. For example, from a Fourier transform of a fast transient, which was recorded with Configuration B (Fig. 8), the resonant frequency of this assembly was determined as 6-7 MHz. Photoexcitation with light modulated at the resonance frequency leads to an amplified response, while the phase angle between the modulated signal and reference waveforms may be used to determine the time-constants.

Finally, we note that the waveguide that contains the FBG may in itself be used as a support for a solid sample, while at the same time delivering not only the probe laser light but also the photoacoustic excitation pulse.

\section{Acknowledgements}

The authors thank Mark Parnis and Scott Hopkins for many helpful discussions. Funding by the Defense Research and Development Canada (Technology Investment Fund, Contract No. W7702-05R068), with minor financial contributions the Canadian Institute for Photonic Innovations, and the Natural Sciences and Engineering Research Council Canada is gratefully acknowledged. We also thank the referees for their insightful comments regarding the deconvolution procedure.

\section{References}

[1] K.O. Hill, Y. Fujii, D.C. Johnson, B.S. Kawasaki, Appl. Phys. Lett. 32 (1978) 647.

[2] K.O. Hill, G. Meltz, J. Lightwave Technol. 15 (1997) 1263.

[3] A.D. Kersey, M.A. Davis, H.J. Patrick, M. LeBlanc, K.P. Koo, C.G. Askins, M.A. Putnam, E.J. Friebele, J. Lightwave Technol. 15 (1997) 1442.

[4] A. Othonos, Rev. Sci. Instrum. 68 (1997) 4309.

[5] Y.J. Rao, Meas. Sci. Technol. 8 (1997) 355.

[6] A. Minardo, A. Cusano, R. Bernini, L. Zeni, M. Giordano, IEEE Trans. Ultrasonics Ferroelectr. Frequ. Contr. 52 (2005) 304.

[7] B. Ortega, D. Pastor, J. Mora, J. Capmany, M.V. Andres, Eurasip. J. Appl. Sig. Process 2005 (2005) 1462.

[8] D. Grobnic, S.J. Mihailov, C.W. Smelser, H.M. Ding, IEEE Photonic Technol. L 16 (2004) 2505.

[9] E. Rivera, D.J. Thomson, Smart Mater. Struct. 15 (2006) 325.

[10] V. Bhatia, A.M. Vengsarkar, Opt. Lett. 21 (1996) 692.

[11] H. Tsuda, Compos. Sci. Technol. 66 (2006) 676.

[12] C.K.N. Patel, A.C. Tam, Rev. Mod. Phys. 53 (1981) 517.

[13] A.C. Tam, Rev. Mod. Phys. 58 (1986) 381.

[14] S.E. Braslavsky, G.E. Heibel, Chem. Rev. 92 (1992) 1381

[15] S. Leytner, J.T. Hupp, Chem. Phys. Lett. 330 (2000) 231.

[16] N. Takahashi, A. Hirose, S. Takahashi, Opt. Rev. 4 (1997) 691.

[17] N. Takahashi, K. Yoshimura, S. Takahashi, K. Imamura, Ultrasonics 38 (2000) 581.

[18] N.E. Fisher, D.J. Webb, C.N. Pannell, D.A. Jackson, L.R. Gavrilov, J.W. Hand, L. Zhang, I. Bennion, Electron. Lett. 34 (1998) 1139.

[19] N.E. Fisher, D.J. Webb, C.N. Pannell, D.A. Jackson, L.R. Gavrilov, J.W. Hand, L. Zhang, I. Bennion, Appl. Opt. 37 (1998) 8120.

[20] W.F. Liu, I.M. Liu, L.W. Chung, D.W. Huang, C.C. Yang, Opt. Lett. 25 (2000) 1319

[21] W.F. Liu, P.S.J. Russell, L. Dong, Opt. Lett. 22 (1997) 1515. 
[22] J.M. Park, S.I. Lee, O.Y. Kwon, H.S. Choi, J.H. Lee, Compos. A: Appl. Sci. Manufact. 34 (2003) 203.

[23] D.C. Betz, G. Thursby, B. Culshaw, W.J. Staszewski, Smart Mater. Struct. 12 (2003) 122.

[24] H. Tsuda, N. Toyama, K. Urabe, J. Takatsubo, Smart Mater. Struct. 13 (2004) 719.

[25] B. Sorazu, G. Thursby, B. Culshaw, F. Dong, S.G. Pierce, Y. Yang, D. Betz, Strain 39 (2003) 111.

[26] M.W. Hathaway, N.E. Fisher, D.J. Webb, C.N. Pannell, D.A. Jackson, L.R. Gavrilov, J.W. Hand, L. Zhang, I. Bennion, Opt. Commun. 171 (1999) 225.

[27] R.B. Wagreich, W.A. Atia, H. Singh, J.S. Sirkis, Electron. Lett. 32 (1996) 1223.

[28] R. Gafsi, M.A. El Sherif, Opt. Fiber Technol. 6 (2000) 299.
[29] C.J.S. de Matos, P. Torres, L.C.G. Valente, W. Margulis, R. Stubbe, J. Lightwave Technol. 19 (2001) 1206.

[30] A.P. Zhang, B.O. Guan, X.M. Tao, H.Y. Tam, Opt. Commun. 206 (2002) 81.

[31] P. Torres, L.C.G. Valente, Opt. Commun. 208 (2002) 285.

[32] J.X. Zhao, X. Zhang, Y.Q. Huang, X.M. Ren, Opt. Commun. 229 (2004) 203.

[33] A.C. Tam, C.K.N. Patel, Appl. Phys. Lett. 35 (1979) 843.

[34] J.E. Rudzki, J.L. Goodman, K.S. Peters, J. Am. Chem. Soc. 107 (1985) 7849.

[35] J.R. Small, L.J. Libertini, E.W. Small, Biophys. Chem. 42 (1992) 29.

[36] P. Fomitchov, S. Krishnaswamy, Opt. Eng. 42 (2003) 956.

[37] T. Erdogan, J. Lightwave Technol. 15 (1997) 1277. 